\title{
Controlled study of malathion and $d$-phenothrin lotions for Pediculus humanus var capitis-infested schoolchildren
}

\author{
Olivier Chosidow, Claude Chastang, Caroline Brue, Elisabeth Bouvet, Mohand Izri, Nicole Monteny, \\ Sylvie Bastuji-Garin, Jean-Jacques Rousset, Jean Revuz
}

\section{Summary}

Anecdotal reports have suggested that reduced efficacy of pediculicides against Pediculus humanus capitis could be related to resistance to treatments.

Ovicidal and pediculicidal activities of $0.5 \%$ malathion and $0.3 \% d$-phenothrin lotions were tested in an experimental model of $P$ humanus capitis grown on rabbits to ensure that the two' treatments were pharmacologically equipotent. We then did a randomised controlled trial in which the lotions were administered to $193 P$ humanus capitisinfested schoolchildren (malathion, 95; d-phenothrin, 98). Success rate was defined as the absence of both live lice and viable nits. Before treatment, live lice were collected and subjected to a pediculicidal test. Pharmacological tests showed $100 \%$ killing of the rabbit-grown nits and lice after exposure to both pediculicides. On day 1 of the controlled trial, the success rate was $92 \%$ in the malathion group ( $95 \% \mathrm{Cl}, 0.86-0.97$ ) and $40 \%$ in the $d$-phenothrin group (0.30-0.49) ( $p<0.0001)$; on day 7 , it was $95 \%$ in the malathion group $(0.90-0.99)$ and $39 \%$ in the $d$-phenothrin group $10.29-0.48$, $p<0.0001)$. Malathion was also significantly more active in the pediculicidal tests compared to $d$-phenothrin and control.

These results suggest an acquired resistance to d-phenothrin in the schoolchildren tested, since all other conditions of the administration of insecticides were standardised.

Lancet 1994; 344: 1724-27

Service de Médecine Interne, Hôpital de la Salpêtrière, 47 Boulevard de l'Hôpital, 75013 Paris, France (O Chosidow MD, $C$ Brue MD); Départment de Biostatistique et Informatique Médicale, Hôpital Saint-Louis, Paris (C Chastang MD); Service des Maladies Infectieuses, Hôpital Bichat, et Inspection Générale de la Protection Sanitaire, Paris (E Bouvet MD); Laboratoire de Parasitologie, Hôpital Avicenne, Bobigny (M A Izri MD, J J Rousset MD); Laboratoire de Lutte contre les Insectes Nuisibles, ORSTOM, Montpellier (N Monteny PhD); Département de Santé Publique (S Bastuji-Garin MD); and Service de Dermatologie, Hôpital Henri Mondor, Crétell, France (J Revuz MD)

Correspondence to: Dr Chosidow

\section{Introduction}

Pediculosis capitis affects millions of children worldwide, especially those 5-14 years.' Acetylcholinesteraseinhibiting malathion, pyrethrin, and synthetic pyrethroid pediculicides, such as $d$-phenothrin, are widely used treatments. ${ }^{2,3}$ However, an increasing rate of head-louse infestation has been reported in France, USA, Israel, and Turkey and could be related to a reduced efficacy of these treatments. ${ }^{48}$ Several factors may influence the effectiveness of treatments, making interpretation of trial results difficult: ovicidal and pediculicidal activities of the pharmacological formulations, lotions eradicating head lice better than shampoos, ${ }^{9}$ duration and quantity of the preparations applied, frequency. of applications, and reinfestation. Development of clinical and/or parasitological resistance to insecticides has also been suspected of decreasing efficacy, but reports are anecdotal..$^{5,10-13}$ Since a pediculicide should be equally effective against live lice and viable nits, the efficacy of the treatments must be judged both clinically and experimentally. ${ }^{2,3}$

Our study aimed to compare the efficacies of malathion and $d$-phenothrin lotions. We first evaluated the ovicidal and pediculicidal activities of the lotions with Pediculus humanus capitis grown on rabbits to ensure that the two treatments were pharmacologically - equipotent. Afterwards, we did a controlled trial including a parasitological test to assess resistance to the pediculicides.

\section{Patients and methods}

\section{Experimental rabbit model}

$0.5 \%$ malathion lotion (Prioderm, Sarget) and $0.3 \%$ $d$-phenothrin lotion (Item, Gandhour) were tested on P humanus bred on white New Zealand rabbits. ${ }^{14}$ Experimentally-grown lice have never been exposed to pediculicides, thereby preventing them from developing drug resistance. The investigator who graded these tests was unaware of treatment assignments.

Ovicidal test. 130-160 nits, firmly fixed to their support, were immersed in each lotion. After $3 \mathrm{~min}$ of contact, nits were washed three times with tap water, allowed to dry at room temperature, then incubated at $28^{\circ} \mathrm{C}$ with relative humidity of $70-80 \%$. Live-hatched first-instar lice, capable of feeding on blood, were counted. The other nits were nonviable. A group of untreated nits was used as a control.

Pediculicidal test. Two groups of lice of different ages (50 larval instar lice and 50 adults), fed four hours before the test, were immersed in each lotion. After $3 \mathrm{~min}$ of contact, the lice were washed with tap water and dried on filter paper. They were then incubated at $28^{\circ} \mathrm{C}$ with relative humidity of $70-80 \% .24$ hours later, the numbers of live and killed lice were recorded. A third group of lice (same number and same age), which were untreated but washed and dried, served as the control.

\section{Controlled trial}

Enrollment of children. The study protocol was approved by an appropriate institutional review committee and conducted in Paris elementary schools selected because of the high rate of infestation the year before. Children and their parents were 


\begin{tabular}{|c|c|c|c|}
\hline Test & Malathion & d-phenothrin & Control \\
\hline $\begin{array}{l}\text { Ovicidal (nits) } \\
\text { Pediculicidal (lice) }\end{array}$ & $\begin{array}{l}100 \%(n=142) \\
100 \%(n=100)\end{array}$ & $\begin{array}{l}100 \%(n=157) \\
100 \%(n=100)\end{array}$ & $\begin{array}{r}23 \%(n=136) \\
0 \%(n=100)\end{array}$ \\
\hline
\end{tabular}

Table 1: Rabbit-bred $P$ humanus: ovicidal ( $\%$ nonviable nits) and pediculicidal (\% lice killed) activity of malathion and d-phenothrin lotions

unaware of the day the treatment would be given. Eligible children had to be $P$ humanus capitis-infested, have live lice and/or more than 5 creamy white or yellow nits closer than 1.5 $\mathrm{cm}$ to the scalp (the previously reported definition of clinically viable nits $\left.{ }^{15}\right)$, and to have obtained parental written informed consent. A semi-quantitative evaluation of the infestation was made at the time of inclusion: live lice, 0 (absence), $+(i-5),++$ $(6-10),+++(11-20),++++(>20)$. Viable nits (counted on the most severely infested hair clump): 0 (absence), $+(1$ viable nir every 1000 hairs $),++(1$ viable nit every 100 hairs $)$, $+++(1$ viable nit every 10 hairs $),++++(1$ viable nit on every hair). Children were excluded if they had any scalp infection.

Design and procedures. The study was preceded by a training period for physicians and health-care assistants. A psychologist explained the study to the teachers and the children, and distributed an information sheet for the parents written in French or appropriate foreign languages. Since each school class constitutes an environmental unit for $P$ humanus capitis dissemination, the randomisation unit was the class and not the child; all infested children of the same class received the same lotion applied by a health-care assistant not involved in assessing the effect of treatment. This procedure assured a blind assessment of the endpoints. Randomisation was stratified by school. $0.5 \%$ malathion and $0.3 \% d$-phenothrin lotions were from the same lots used in the experimental model. At the time of inclusion, day 0 ; after 24 hours, day 1 ; and 7 days later, day 7; children were examined by an investigator unaware of the lotion used. In another room, health-care assistants applied sufficient lotion to wet the scalp and base of the hair shaft; lotion remaining was kept until the end of the study, then measured to estimate the amount used. Lotion was applied once in the morning. Any other pediculicidal treatment was forbidden during the study. Since $d$-phenothrin has to be applied for at least 10 minutes and malathion for at least 6 hours, ${ }^{2}$ we instructed the parents to rinse their children's hair with a standard non-treating shampoo (to remove the smell) only after 8-12 hours; we further instructed them to treat sheets, pillowcases, clothes, hats, soft toys, or other fomites with an appropriate pediculicidal powder. Children's contacts, eg, family members, received instructions to treat themselves with the same lotion as the child.

Parasitological evaluation. Activities of lotions were evaluated on human-grown lice in a hatching-nits test and a pediculicidal test. 15,16 Nits were collected on day 0 and, when infestation persisted, on day 1 and day 7 ; lice were collected on day 1 . Hairs with at least 5 viable nits were removed with scissors. Lice were removed with a fine-tooth comb. Nits and lice were transferred to sterile Petri dishes, closed with self-adhesive tape, identified, and quickly transported to the parasitology laboratory. The parasitologist was unaware of the insecticide assignments for both tests.

Hatching-nits test. Nits were examined with a binocular microscope; empty or probably dead nits were discarded and plump nits transferred to another Petri dish. Dishes were incubated at $29^{\circ} \mathrm{C}$ with relative humidity of $70-80 \%$, and examined twice daily for 10 days. Hatched nits were judged viable if the young lice were able to feed on the back of the hand of one of us (MI) in less than 20 minutes; the other nits were considered nonviable.

Pediculicidal tests. Lice were examined with a binocular microscope; dead, damaged, or less than fully mobile lice were discarded. Pediculicidal lotion was applied acording to WHO recommendations to determine susceptibility. ${ }^{13,17}$ This pediculicidal test was first validated by a preliminary study with the experimental model of $P$ humanus capitis bred on rabbits (unpublished data).

\section{End points}

On day 1 and day 7 , the absence of both live lice and parasitologically viable nits (hatching test) defined the success of treatment; the main end point of the trial was the success rate on day 7. Live lice and viable nits were also compared between groups.

\section{Analysis}

Efficacy of malathion and $d$-phenothrin lotions is between 95-100\%.,18 An occasional report suggested a lower efficacy of these treatments, therefore we chose a day 7 success rate of $90 \%$. Sample size was estimated at 376 (188 in each group) on an assumption of benefit given by a success rate on day 7 of $85 \%$ with $d$-phenothrin and $95 \%$ with malathion, a type I error of 0.05 , a type II error of 0.10 , and a two-sided test. Given an infestation rate of $5-10 \%$, screening of 8400 children was foreseen. One interim analysis was planned at a level of $\alpha^{\prime}=0.029$ to maintain an overall level of $\alpha=0.05$.

Analysis was on intention-to-treat. We used non-parametric tests 'Fisher's test for binary parameters, $\chi^{2}$ for categorical parameters and the Kruskal and Wallis test for continuous parameters) to compare treatment groups. Treatment comparison was adjusted for categorical covariates with the Mantel-Haenszel test: Since the randomisation unit was the schoolclass and not the child, a generalised regression model provided an appropriate test for comparing the two treatment groups. 95\% CIs were computed for the main end point.

Louse survival from the pediculicidal test on human-grown $P$ humanus were analysed with Kaplan-Meier estimates and logrank tests.

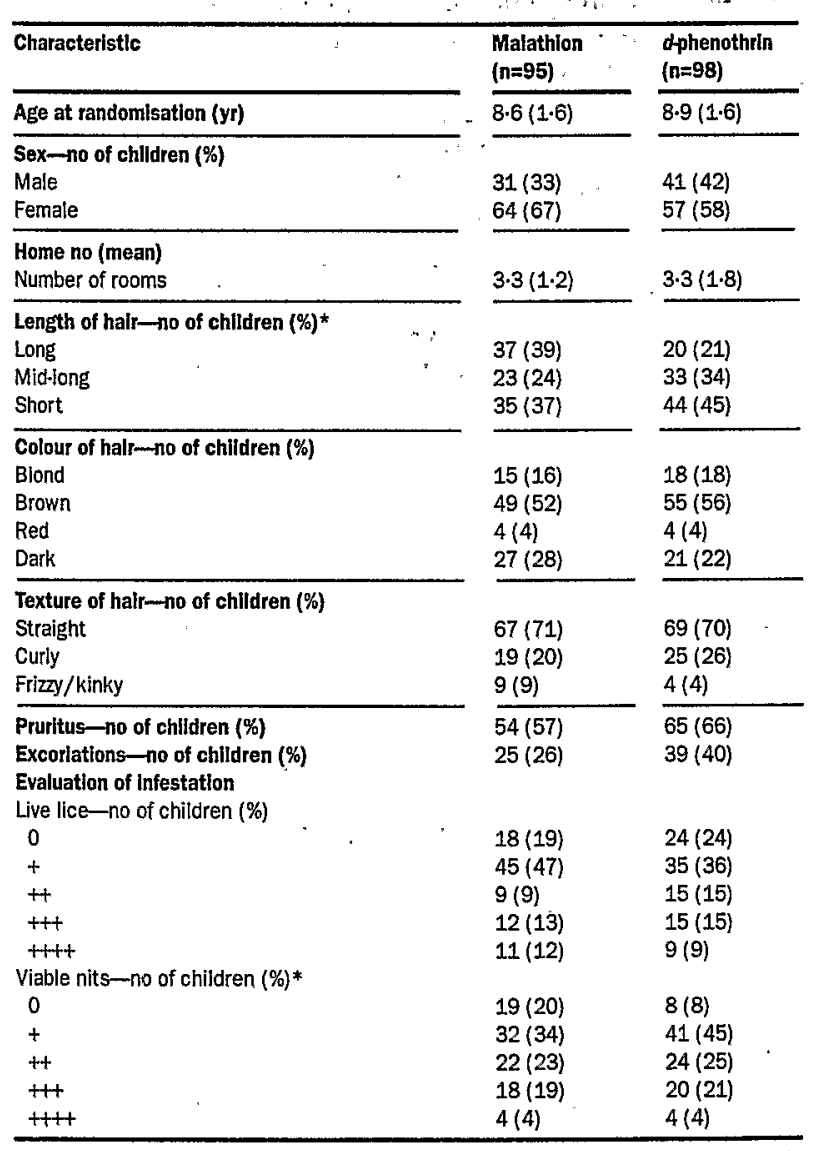

The 2 groups were similar at baseline except for a significant difference for the length of hair ( $p=0.02$; chi-square). * 0 ne value missing in the $d$-phenothrin group.

Table 2: Baseline characteristics of the $\boldsymbol{P}$ humanus capitisinfested schoolchildren assigned to receive malathion or aphenothrin lotion* 


\section{Results}

Table 1 compares insecticidal activities of malathion and $d$-phenothrin lotions assessed in the experimental model of rabbit-grown $P$ humanus. There was complete ovicidal and pediculicidal activity (100\% mortality of nits and lice after contact with either lotion).

The controlled trial took place January-May, 1992. 8353 children were screened; 279 were infested. Parental written informed consent was obtained for 193 children from 137 classes which were randomised to receive either malathion lotion or $d$-phenothrin lotion. The number of infested children in the classes was: 1 child in 93, 2 in 33, 3 in 10 , and 4 in 1.95 children received malathion lotion and 98 received $d$-phenothrin lotion. Baseline characteristics were similar except for a significant difference for the length of hair in the $d$-phenothrin group $\left(p=0.02 ; x^{2}\right)$ (table 2$)$. Based on the total volumes of insecticidal lotions remaining after treatment of 81 children in the malathion group and 82 children in the $d-$ phenothrin group, amounts applied in the two groups were similar (mean $17.2 \mathrm{~mL}$ vs $16.2 \mathrm{~mL}$ ). All but 7 children were seen at the scheduled visits: 3 missed the visit on day 1 ( 1 in the malathion group and 2 in the $d$ phenothrin group) and 4 others ( 1 in the malathion group and 3 in the $d$-phenothrin group), seen on day 1 , were lost to follow-up by day 7 ; we were able to estimate the missing values based on the observations noted at the other visit.

On day 1 , the success rate was $92 \%$ in the malathion group and $40 \%$ in the $d$-phenothrin group ( $p<0.0001$; Fisher's test and generalised regression model) (table 3). On day 7 , malathion's success rate was $95 \%$ and that of the $d$-phenothrin group was $39 \%(\mathbf{p}<0 \cdot 0001)$. Adjusting for the length of hair and the distribution of live lice and viable nits in the comparison of treatment groups did not modify the results ( $p<0.0001 ;$ Mantel-Haenszel test). On day 7 , live lice were found in 6 malathion-treated children and in $58 d$-phenothrin-treated children. Among the 87 malathion-treated children considered to be cured on day $1,4(5 \%)$ were not cured on day 7 and, among the 8 children considered not to be cured on day 1, 1 (13\%) remained infested on day 7 . Among the $39 d$-phenothrintreated group considered to be cured on day $1,14(36 \%)$ were not cured on day 7 , and among the 59 children considered not to be cured on day $7,46(78 \%)$ remained infested on day 7. No side effects were reported. Interim

\begin{tabular}{|c|c|c|c|}
\hline Day & Malathion & d-phenothrin & p \\
\hline 1 & $92 \%(0.86-0.97)$ & $40 \%(0.30-0.49)$ & $<0.0001$ \\
\hline 7 & $95 \%(0.90-0.99)$ & $39 \%(0.29-0.48)$ & $<0.0001$ \\
\hline
\end{tabular}

Table 3: Success rates in schoolchildren assigned to receive malathion or $\alpha$-phenothrin lotion

\begin{tabular}{|c|c|c|c|}
\hline Test & $\begin{array}{l}\text { Malathion } \\
(n=357)\end{array}$ & $\begin{array}{l}\text { d-phenothrin } \\
(n=416)\end{array}$ & $\begin{array}{l}\text { Control } \\
(n=320)\end{array}$ \\
\hline \multicolumn{4}{|l|}{ Dead at } \\
\hline $1 \mathrm{~h}$ & $357(100 \%)$ & $40(9.6 \%)$ & $22(6.9 \%)$ \\
\hline $2 h$ & - & $12(2.9 \%)$ & $9(2.8 \%)$ \\
\hline $24 \mathrm{~h}$ & - & $282(67 \cdot 8 \%)$ & $245(76 \cdot 6 \%)$ \\
\hline Total & $357(100 \%)$ & $334(80.3 \%)$ & $276(86 \cdot 3 \%)$ \\
\hline $\begin{array}{l}\text { Alive at } \\
24 \mathrm{~h}\end{array}$ & 0 & $82(19.7 \%)$ & $44(13 \cdot 7 \%)$ \\
\hline
\end{tabular}

Table 4: Pediculicidal test (survival distribution) at 3 different times after application of malathion, d-phenothrin, or no treatment to live lice collected from the heads of infested schoolchildren results led to the decision to stop the study early, in accordance with the protocol $\left(\alpha^{\prime}=0.029\right)$.

Results of the parasitological rests were used to compare the survival distributions of live lice collected from the heads of schoolchildren and subjected to malathion, $d$-phenothrin, or no treatment (control group) in the laboratory (table 4). One hour after application, $100 \%$ of the lice had been killed by malathion and $9.6 \%$ by $d$-phenothrin as compared with $6.9 \%$ of the control. Two hours after application, $d$-phenothrin had killed $12.5 \%$ of the lice, while $9.7 \%$ of the control lice were dead. 24 hours after application, $80.3 \%$ of the $d$-phenothrin-treated lice were dead os $86.3 \%$ of the control. Survival distributions were significantly different between the three groups ( $p<0.001$; log-rank test), with the survival distribution of the malathion group being different from both $d$-phenothrin and control groups ( $<<0.001$; log-rank test). No difference was found between $d$-phenothrin and the control group $(p=0.33$; log-rank test).

\section{Discussion}

We found that a single application of malathion was more effective, evaluated as the percentage of children without both live lice and parasitologically viable nits, than $d$-phenothrin. As eggs need about a week to hatch, the main end point of the trial was set at day 7 . Before the trial, we hypothesised that a $10 \%$ difference between the two insecticides would be clinically relevant. Indeed, malathion showed a better ovicidal efficacy, as indicated by the success rate on day 7 (95\% vs $39 \%$ ) but also a better pediculicidal efficacy, as indicated by the success rate on day $1(92 \%$ vs $40 \%)$, regardless of the extent of infestation. Moreover, patients cured on day 1 in the $d$-phenothrin group had a $36 \%$ probability of having a relapse on day 7 , while $95 \%$ of the patients cured on day 1 in the malathion group remained cured on day 7. Because viable nits are sometimes difficult to recognise clinically, we did a nithatching test to identify parasitologically viable nits, ${ }^{15}$ before any insecticidal treatment was applied. The results confirmed the clinical observation that malathion was better, killing all the live lice, regardless of the duration of the application, unlike $d$-phenothrin and untreated controls.

Several factors may influence the efficacy of pediculicidal treatments. Before starting the trial, we confirmed that the commercially available malathion and $d$-phenothrin lotions were equally effective in eradicating nits and head lice bred on rabbits. We used a lotion preparation, which is the best for eradicating head lice. ${ }^{9}$ The characteristics of both groups were similar for the duration of application and volume of lotion used, number of rooms at home that can be considered as a measure of contact or transmission, and hair colour and texture. $D$-phenothrin was applied for at least 8 hours which could have increased the efficacy of this treatment, since the usual duration of pyrethrin and pyrethroid application is 10 to 60 minutes. ${ }^{2,3}$ The distribution of live lice and viable nits and, consequently, the extent of lice infestation was also similar in both groups. Although the two groups differed for the length of hair at the time of inclusion, the conclusions remained unchanged when the analysis was adjusted for this parameter. Moreover, no direct correlation between the length of hair and lice infestation rates has been shown. ${ }^{19}$ Reinfestation was not a 
bias in our trial, as the study was controlled and the parents were instructed to treat all the family members and the fomites at the same time as the index case.

Ovicidal and pediculicidal activities of the formulation and also important. Preliminary pharmacological tests on rabbit-bred lice and nits indicated that malathion and $d$-phenothrin had excellent activities. Because $d$-phenothrin is a synthetic pyrethroid with a greater specific pediculicidal activity than natural pyrethrins, ${ }^{2}$ we did not use a mixture containing piperonyl butoxide, a synergist for pyrethrins. Commercially available products were chosen to evaluate the efficacy of insecticides on live lice. Although the results differed somewhat, the pediculicidal test on human-grown head lice reflected the conclusions of the clinical trial. A similar model using freshly collected head lice has previously been described and could represent an alternative approach. ${ }^{16}$

The repeated use of one pediculicide on a louse population is likely to select resistant lice. ${ }^{20,21} P$ humanus capitis resistance has already been reported. Reports of resistance to DDT and lindane led to the introduction of carbaryl, malathion, pyrethrins, and synthetic pyrethroids. ${ }^{10,13,22}$ Resistance to these new insecticides has also been reported but remains anecdotal. ${ }^{11-13}$ We found malathion and $d$-phenothrin to be equipotent in an experimental model of $P$ humanus bred on rabbits. Although this species differs in its feeding habits, lifestyle, and appearance from human head lice, the model was thought to be predictive of the pediculicidal activity in human louse infestation, ${ }^{14,16}$ although these experimental lice were never exposed to pediculicides other than during testing and thus their development of resistance was prevented. It appears that results in laboratory-raised lice must not be predictive of results in children with pediculosis capitis.

Our results suggest the emergence of acquired resistance to this treatment by the tested head lice, since conditions of insecticide administration were standardised. In the UK, a trial showed a slightly greater efficacy of a $0.2 \% d$-phenothrin shampoo vs a $0.5 \%$ malathion lotion, ${ }^{18}$ a discrepancy that could reflect the over-use of pediculicides selecting resistant head-lice, especially in countries where pediculicides have been used for the past 10-20 years..$^{13}$ Malathion was marketed in France in 1983, whereas it entered the British market in $1971,{ }^{2}$ unlike the pyrethrins and synthetic pyrethroids that were developed in France 15 years ago. Moreover, the latter are widely used in many countries because they are safe, photostable, cosmetically more acceptable, easy to use, and may be prophylactic. ${ }^{2,3}$ Shampoos expose lice to a short contact time and a low concentration of insecticide which may favour resistance to treatment..23,24

Regardless of the mechanism of resistance, physicians and pharmacists should be made aware of possible variations in the efficacy of pediculicidal preparations, and should advise non-responders to switch to another type, for example from pyrethrins or pyrethroids to malathion. The possibility of incomplete ovicidal activity may also lead to a standard recommendation of a second treatment after 7-10 days. New formulations and concentrations of insecticidal products, such as $1 \%$ permethrin or an association of pyrethrins or pyrethroids with malathion, would perhaps achieve better control of head-louse infestation. ${ }^{3}$ In the UK, the policy of alternating the use of malathion and carbaryl every three years has been thought to minimise the risk of developing resistance. ${ }^{25}$
We think that it is always advisable to introduce new insecticides within this public-health policy to reduce the risk further. A regional survey based on a periodic pediculicidal test could also help to select the best pediculicide to eradicate $P$ humanus capitis.

We thank J M Alexandre, Y Benin, L Blum, N Bravard, J Brice, C Brière, M Carnec, C Caulin, A Chauvin, J Choin, L Chosidow, C Cotel, F Ficat, L Fonteneau, C Gervais-Philippe, S Guiguen, J C Guillaume, $S$ Herson, B Hubert, J Jacobson, T Kalo, M F Kogut, Y Layer, Y Leclerc, A

Mansion, C Muziot, C Paciot', F Poli, R Pomarede, M D Riou, M Robert, G Rykner, V Saada, N Sade, E Serna, A Serra for help in the study. We are inciebred to all the children we visited at school and their parents, to the medical staff, social workers, teachers and school principals.

This work was supported by grants from the Direction Générale de la Santé and the Direction de la Pharmacie et du Médicament, Ministère de la Santé, the Délégation à la Recherche Clinique, Assistance PubliqueHôpitaux de Paris (911409), and the Institut National de la Santé et de la Recherche Médicale (INSERM) (CNEP 91CN51). Sarget Drug

Company provided malathion (Prioderm) free of charge.

\section{References}

1 Gulati $P$, Kamat J, Singh P. A community based epidemiological study of louse infestation. Clinician 1981; 45: 177-81.

2 Burns DA. The treatment of human ectoparasite infection. $\mathrm{Br} \mathcal{F}$ Dermatol 1991; 125: 89-93.

3 Taplin D, Meinking TL. Pyrethrins and pyrethroids in dermatology. Arch Dermatol 1990; 126: 213-21.

4 Anonymous. Head lice in the seventies. Lancet 1979; ii: 19-21.

5 Combescot $\mathrm{C}$. Epidémiologie actuelle de la pédiculose à Pediculus capitis. Bull Acad Natl Med (Paris) 1990; 174: 231-37.

6 Gratz NG. Epidemiology of louse infestations. In: Orkin M, Maibach HI, eds. Cutaneous infestations and insects bites. New York: Praeger, 1983: $151-63$.

7 Mumcuoglu KY, Miller J, Gogin R, et al. Epidemiological studies on head lice infestation in Israel. Int $\mathcal{f}$ Dermatol 1990; 29: 502-06.

8 Ünal $\mathrm{G}$, Kutlar $M$, Onsun N. Pediculosis capitis in Istanbul. Int I Dermatol 1993; 32: 30-32.

9 King F, Lewis S, Robert C. Head lice: questions and answers. Pharm $f$ 1988; 241: 667-68.

10 Maunder JW. Resistance to organochlorine insecticides in head lice, and trials using alternative compounds. Med Officer 1971; 125: 27-29.

11 Miller RN, Wisseman CL, Sweeney GW, Verschueren A, Fabrikant IB. First report of resistance of human body lice to malathion. Trans $R$ Soc Trop Med Fyg 1972; 66: 372-75.

12 Cole MM, Clarck PH, Washington F, Ellerbe W, Van Natta DL. Resistance to malathion in a strain of body lice from Burundi. I Econ Entomol 1973; 66: 118-19.

13 Rasmussen JE. Pediculosis: treatment and resistance. In: Callen JP, Dahl MV, Golitz LE, eds. Advances in dermatology vol 1. Chicago: Year Book Medical Publishers, 1986: 109-25.

14 Valade M. Le pou de l'homme, Pediculus humanus Linné, 1758. Observations biologiques. Evaluation de l'activité de diffërents insecticides. Paris, France: Editions de l'ORSTROM, 1985, PhD thesis.

15 Taplin D, Castillero PM, Spiegel J, Mercer S, Rivera AA, Schachner L. Malathion for treatment of Pediculus humanus var capitis infestation. IAMA 1982; 247: 3103-05.

16 Meinking TL, Taplin D. Comparative efficacy of treatment for pediculosis capitis infestation. Arch Dermatol 1986; 122: 267-71.

17 WHO. Résistance des vecteurs de maladies aux pesticides; cinquième rapport du comité OMS d'experts de la biologie des vecteurs et de la lutte antivectorielle. Rapport technique no 655, 1981.

18 Kyle $R$. Comparison of phenothrin shampoo and malathion lotion in the treatment of head louse infection. $f R$ Soc Health 1990; 110: 62-63.

19 Maunder WW. The appreciation of lice. Proc R Inst Great Britain 1983; 55: $1-31$.

20 Cole MM, Clark PH. Development of resistance to synergized pyrethrins in body lice, and cross resistance to DDT. $\mathcal{F}$ Econ Entomol 1961; 54: 649-51.

21 Pasteur N, Raymond M, Pauplin Y, Nance E, Heyse D, Mouches C. Role of gene amplification in insecticide resistance. In: Casida JE, ed. Pesticides and alternatives. Amsterdam: Elsevier Science Publishers, 1990: 439-77.

22 Kucirka SA, Parish LC, Witkowski JA. The story of lindane resistance and head lice. Int $\mathcal{J}$ Dermatol 1983; 22: 551-52.

23 Fine BC. Pediculosis capitis. $N$ Engl F Med 1983; 309: 1461.

24 Anonymous. Napp launches new head lice shampoo amid criticism. Pharm f 1990; 244: 263.

25 Doss S, Powell CA, Miller AJ. Phenothrin lotion, the latest recruit in the battle against head lice: the results of two controlled comparative studies. F R Soc Healch 1991; 111: 47-50. 